OPEN ACCESS

Edited by:

Jun Izawa,

University of Tsukuba, Japan

Reviewed by:

Hidenori Aizawa,

Hiroshima University, Japan Satoshi Kodama,

The University of Tokyo, Japan

*Correspondence:

Daisuke Ishii

ishiid@ipu.ac.jp

Received: 22 April 2021 Accepted: 26 July 2021 Published: 17 August 2021

Citation:

Ishii $D$, Ishibashi $K$, Yuine $H$, Takeda K, Yamamoto S, Kaku Y,

Yozu A and Kohno Y

(2021) Contralateral and Ipsilateral Interactions in the Somatosensory

Pathway in Healthy Humans. Front. Syst. Neurosci. 15:698758. doi: 10.3389/fnsys.2021.698758

\section{Contralateral and Ipsilateral Interactions in the Somatosensory Pathway in Healthy Humans}

\author{
Daisuke Ishii ${ }^{1,2 *}$, Kiyoshige Ishibashi ${ }^{3}$, Hiroshi Yuine ${ }^{4}$, Kotaro Takeda $^{5}$, \\ Satoshi Yamamoto ${ }^{6}$, Yuki Kaku ${ }^{1}$, Arito Yozu ${ }^{1}$ and Yutaka Kohno ${ }^{1}$
}

${ }^{1}$ Center for Medical Sciences, Ibaraki Prefectural University of Health Sciences, Ami, Japan, ${ }^{2}$ Department of Cognitive Behavioral Physiology, Chiba University Graduate School of Medicine, Chiba, Japan, ${ }^{3}$ Department of Physical Therapy, Ibaraki Prefectural University of Health Sciences Hospital, Ami, Japan, ${ }^{4}$ Department of Occupational Therapy, School of Health Sciences, Ibaraki Prefectural University of Health Sciences, Ami, Japan, ${ }^{5}$ Faculty of Rehabilitation, School of Healthcare, Fujita Health University, Toyoake, Japan, ${ }^{6}$ Department of Physical Therapy, School of Health Sciences, Ibaraki Prefectural University of Health Sciences, Ami, Japan

Hyper-adaptability, the ability to adapt to changes in the internal environment caused by neurological disorders, is necessary to recover from various disabilities, such as motor paralysis and sensory impairment. In the recovery from motor paralysis, the pre-existing neural pathway of the ipsilateral descending pathway, which is normally suppressed and preserved in the course of development, is activated to contribute to the motor control of the paretic limb. Conversely, in sensory pathways, it remains unclear whether there are compensatory pathways which are beneficial for the recovery of sensory impairment due to damaged unilateral somatosensory pathways, such as thalamic hemorrhage. Here, we investigated the interaction between the left and right somatosensory pathways in healthy humans using paired median nerve somatosensory evoked potentials (SEPS). Paired median nerve SEPs were recorded at CP3 and CP4 with a reference of Fz in the International 10-20 System. The paired median nerve stimulation with different interstimulus intervals (ISIs; 1, 2, 3, 5, 10, 20, 40, 60, and $100 \mathrm{~ms}$ ) was performed to test the influence of the first stimulus (to the right median nerve) on the P14, P14/N20, and N20/P25 components induced by the second stimulus (left side). Results showed that the first stimulation had no effect on SEP amplitudes (P14, P14/N20, and N20/P25) evoked by the second stimulation in all ISI conditions, suggesting that there might not be a neural connectivity formed by a small number of synapses in the left-right interaction of the somatosensory pathway. Additionally, the somatosensory pathway may be less diverse in healthy participants.

Keywords: evoked potentials, somatosensory, corpus callosum, median nerve, humans

\section{INTRODUCTION}

Hyper-adaptability can be defined as the ability to adapt to changes in the internal environment caused by neurological disorders (e.g., stroke and spinal cord injury); it is essential for recovery from various disabilities. Corticospinal neurons in the forelimb region of the primary motor cortex project to contralateral and ipsilateral spinal interneurons (Yoshino-Saito et al., 2010). This ipsilateral descending pathway plays an important role in the recovery from motor paralysis after stroke (Netz et al., 1997; Levy et al., 2001). Moreover, previous studies on monkeys with spinal 
cord-lesions showed that the sprouting of midline-crossing axons of the corticospinal tract occurs in the spinal cord rostral to the lesion, and the sprouting was associated with improvement in hand function and locomotion (Courtine et al., 2005; Rosenzweig et al., 2010). Additionally, functional motor representation maps around the damage and remote cortical regions change with rehabilitative motor training after focal damage in the forelimb movement area of the motor cortex (Nudo et al., 1996; Frost et al., 2003; Ramanathan et al., 2006; Barbay et al., 2013). These studies suggest that a pathway normally suppressed and preserved in the course of development may participate in the control of the paretic limb to adapt to changes in the internal environment (Murata et al., 2015; Isa, 2017, 2019; Isa et al., 2019; Yamamoto et al., 2019; Kato et al., 2020).

A functional magnetic resonance imaging (fMRI) study of sensory pathways revealed that not only the contralateral primary somatosensory cortex (SI) but also the ipsilateral SI was activated following the median nerve stimulation (Nihashi et al., 2005). In macaque monkeys, bilateral receptive fields have been found in the somatosensory area 2, which is considered to be the homolog of Brodmann's area 2 in human SI (Iwamura et al., 2001, 2002). Neural responses in the bilateral receptive fields were not found after making postcentral gyrus lesions in the contralateral hemisphere, suggesting that the neurons in the bilateral receptive fields receive the sensory information from the contralateral brain through interhemispheric transfer (Iwamura et al., 1994). The presence of a left-right interaction at various levels is important for recovery from hypoesthesia because the left-right interaction through the corpus callosum does not contribute to the recovery of hypoesthesia in cases where somatosensory pathways are damaged at the subcortical level, such as the thalamus. Several studies investigated the interactions between contralateral and ipsilateral activations using a paired median nerve somatosensory evoked potential ( $\mathrm{p}$-SEP) protocol (Ragert et al., 2011; Brodie et al., 2014). In this protocol, peripheral stimulation of the unilateral median nerve as conditioning stimulus (CS) always preceded the stimulation of the other median nerve as the test stimulus (TS) with some interstimulus intervals (ISIs). In the presence of a left-right interaction, the SEP evoked by the TS is attenuated by the interference of the CS. The levels of the left-right interaction in the somatosensory pathway were estimated by the length of the ISI (Ragert et al., 2011; Brodie et al., 2014). It is unclear whether the amplitude of SEPs evoked by the second median nerve stimulation is attenuated or eliminated by the first median nerve stimulation in cases of short ISIs, especially in those less than $5 \mathrm{~ms}$. On the other hand, Ragert et al. showed that the first stimulation to the left median nerve decreased the SEP amplitudes (N20) evoked by the second stimulation to the right median nerve with an ISI of 20-25 ms (Ragert et al., 2011). Additionally, Brodie et al. showed that the first stimulation to the right median nerve decreased the SEP amplitudes (P14/N20 and N20/P25) evoked by the second stimulation to the left median nerve with ISIs of 25-35 and 15-35 ms, respectively (Brodie et al., 2014). These studies indicate that interhemispheric inhibitory interactions in the SI occur between the two hemispheres through the corpus callosum in the critical time interval of $20-25 \mathrm{~ms}$ or $15-35 \mathrm{~ms}$ after median nerve stimulation (Ragert et al., 2011; Brodie et al., 2014). Moreover, the results of these two previous studies are inconsistent, and there is no consensus on the ISI interval regarding the left-right interaction of somatosensory pathways. In addition, the commissural fibers between the left and right sides of the hemispheres comprise not only the corpus callosum but also other commissural fibers such as the anterior and posterior commissural fibers. It is necessary to elucidate whether there are somatosensory pathways that provide input to the contralateral SI over a long period of time through these routes. Therefore, $\mathrm{p}$-SEP recordings were obtained at nine different ISIs $(1,2,3,5,10,20,40,60$, and $100 \mathrm{~ms})$ to investigate the interaction between contralateral and ipsilateral activations at various levels in healthy humans.

\section{MATERIALS AND METHODS}

\section{Participants}

Fourteen healthy right-handed males (mean age: 30.6; range: 25-39 years) participated in this study. The total sample size $(n=14)$ was calculated using the $\mathrm{G}^{\star}$ Power 3.1.9.2 software (repeated measures, within factors, F-tests, effect size f: 0.25 , $\alpha$ error: 0.05, Power: 0.8, number of measurements: 10; Faul et al., 2007). No participants had a history of neurological or psychiatric problems. The present study was approved by the Ethics Committee of Ibaraki Prefectural University of Health Sciences (approval no. 893 and e278).

\section{SEP Recording}

To investigate the interactions between contralateral and ipsilateral activations at a level below the brainstem, single median nerve SEPs (s-SEPs) and paired median nerve SEPs ( $\mathrm{p}$ SEPs) were recorded using a previously described protocol with minor modifications (Ragert et al., 2011; Brodie et al., 2014; Figures 1A,B). In the s-SEP paradigm, s-SEPs were obtained by stimulating the right and left median nerve at the wrist. In the p-SEP paradigm, peripheral stimulation of the right median nerve as conditioning stimulus (CS) always preceded the left median nerve stimulation as the test stimulus (TS) with nine different ISIs $(1,2,3,5,10,20,40,60$, and $100 \mathrm{~ms})$. Electroencephalogram (EEG) data from three-channel electrodes (Fz, CP3, and CP4) were recorded using a BrainAmp DC (Brain Products, Gilching, Germany) and TMS-compatible EEG electrode caps. The ground electrode was placed on the skin over the midpoint between the radius and ulna at the height of the proximal $1 / 2$ of the right forearm, and the linked ear was used as a reference. The impedance of each electrode was set at $<5 \mathrm{k} \Omega$. EEG signals were recorded at a sampling frequency of $5 \mathrm{kHz}$ with a bandpass of DC- $1 \mathrm{kHz}$.

A Neuropack X1 (Nihon kohden, Tokyo, Japan) was used to deliver electrical stimuli of $0.2 \mathrm{~ms}$ duration at a rate of $3 \mathrm{~Hz}$. The stimulus intensity was set three times the perceptual threshold $[7.46 \pm 1.21 \mathrm{~mA}$ for the right median nerve, $7.58 \pm 0.52 \mathrm{~mA}$ for the left median nerve (mean \pm standard deviation SD)]. During the $\mathrm{p}$-SEP condition, the right median nerve stimulation always proceeded the left median nerve stimulation using nine different ISIs (1-100 ms). During the s-SEP condition, a test stimulus (TS 

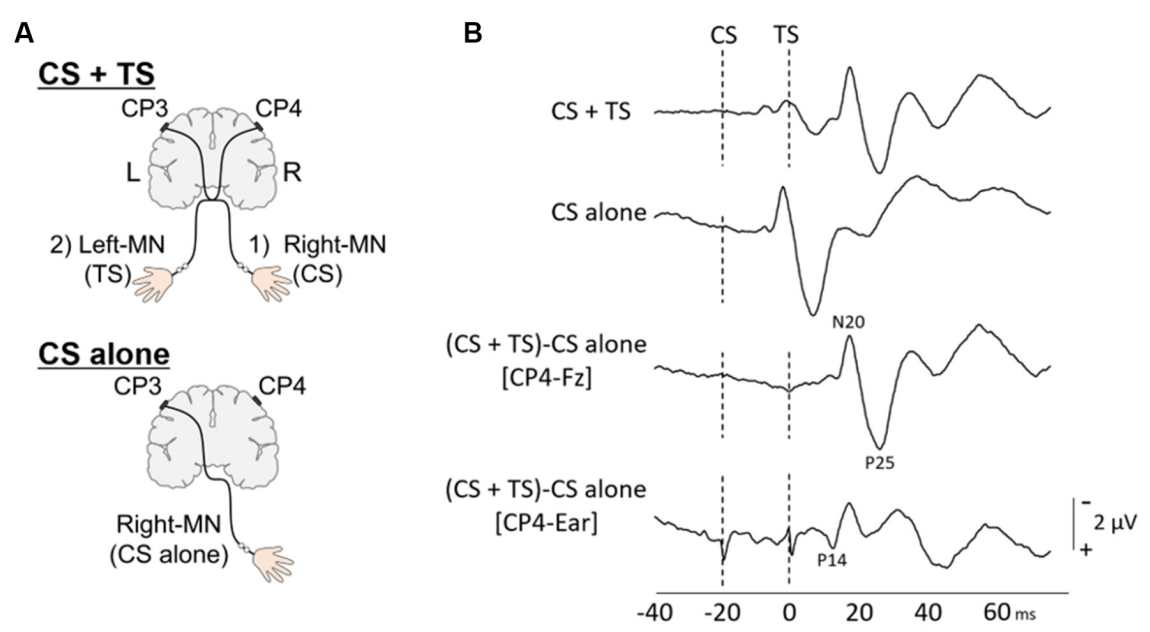

FIGURE 1 | Paired median nerve somatosensory evoked potential ( $p$-SEP). (A) To investigate the effect of conditioning stimulus (CS) on the SEP induced by the test stimulus (TS) (upper panel), the paired median nerve SEPS (p-SEPs) and (lower panel) the single median nerve SEPs (s-SEPs) were recorded at CP4 electrodes. In the p-SEP recording: (1) the right median nerve (right-MN) stimulation as CS always preceded; (2) the left median nerve (Left-MN) stimulation as TS with nine different interstimulus intervals (ISIs; 1, 2, 3, 5, 10, 20, 40, 60, and 100 ms). (B) Typical p-SEP (ISI; 20 ms) and s-SEP waveforms at CP4 from a representative individual. Dotted line: the time of stimulation. The p-SEP data were analyzed using the following formula: $\mathrm{p}-\mathrm{SEPS}=(\mathrm{SEP}$ response of CS + TS at CP4) - (SEP response of CS alone at CP4).

alone, left median nerve) and a control stimulus (CS alone, right median nerve) were applied. The order of conditions [CS + TS (1-100 ms), TS alone, and CS alone] was pseudo-randomized during the experiment. A total of five, 500 stimulus-related epochs were recorded with 500 epochs for each condition [9 ISIs $(\mathrm{CS}+\mathrm{TS})$, TS alone, and CS alone]. We asked the patients to count the electrical stimuli during SEP recordings to counteract the attention effect.

\section{Data Analysis}

Epochs were digitally filtered using a band-pass Butterworth filter $(1-200 \mathrm{~Hz})$, and each condition was averaged (Ragert et al., 2011). Each SEP component was calculated by montages described below using Matlab R2019a (MathWorks, Natick, MA, United States; Mauguiere et al., 1999; Cruccu et al., 2008). The subcortical component $(\mathrm{P} 14)=\mathrm{CP} 3$ or CP4-linked earlobes; the cortical components (P14-peak-N20-peak and N20-peak-P25) = CP3 or CP4 - Fz. Amplitudes of the subcortical component (P14) were measured as the P14-onset to P14-peak. Amplitudes of cortical components (P14-peak-N20peak and N20-peak-P25) were measured from peak to peak.

To evaluate the effect of CS (right median nerve stimulation) on TS (left median nerve stimulation), the p-SEP data were analyzed using the following formula:

p-SEPs at CP3 or CP4 $=(S E P$ response of $C S+T S)-(S E P$ response of CS only)

\section{Statistical Analyses}

All analyses were conducted using the SPSS 12.0 for Windows (SPSS, Chicago, Illinois). Data are shown as mean \pm SD for all results (Figure 2). Data distribution was evaluated using the
Shapiro-Wilk normality test. All data were normally distributed. Data of each amplitude (P14, P14/N20, N20/P25) were analyzed using one-way repeated-measures analysis of variance with factor ISI conditions (TS alone, 1, 2, 3, 5, 10, 20, 40, 60, and $100 \mathrm{~ms}$ ). Dunnett's test was used for post hoc comparisons. Statistical significance was set at $p<0.05$.

\section{RESULTS}

Supplementary Figures 1-14 show the s-SEP and analyzed p-SEP (s-SEP subtracted) waveforms at CP3 and CP4 from all participants. The Shapiro-Wilk test showed that all data were normally distributed $(p>0.05)$. Figures $2 \mathrm{~A}-\mathbf{F}$ show no significant main effects on ISI conditions in all amplitudes at CP3 and CP4 (P14: CP3, $F_{(9,117)}=1.421, p=0.187 ; \mathrm{CP} 4$, $F_{(9,117)}=1.236, p=0.280 ; \mathrm{P} 14 / \mathrm{N} 20: \mathrm{CP} 3, F_{(9,117)}=1.574$, $p=0.131 ; \mathrm{CP} 4, F_{(9,117)}=0.947 ; p=0.487$; and N20/P25: CP3, $\left.F_{(9,117)}=0.730, p=0.681 ; \mathrm{CP} 4, F_{(9,117)}=0.938 ; p=0.495\right)$. Dunnett's test showed no significant differences between each ISI and TS alone condition in all amplitudes at CP3 and CP4 $(p>$ 0.05).

\section{DISCUSSION}

In this study, we investigated the interaction between contralateral and ipsilateral somatosensory pathways in healthy humans. The major findings of this study were that the right median nerve stimulation (CS) did not interfere with SEP amplitude (P14, P14/N20, N20/P25) induced by left median nerve stimulation (TS) in all ISI conditions $(1,2,3,5,10,20,40$, 60 , and $100 \mathrm{~ms})$. 

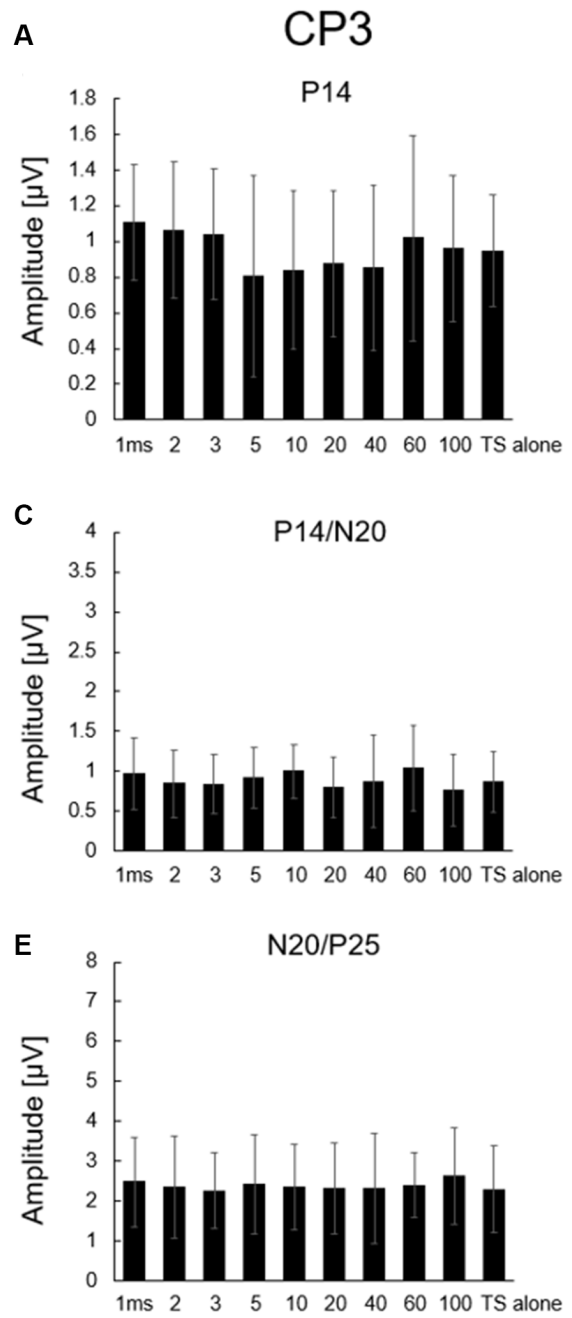

B CP4

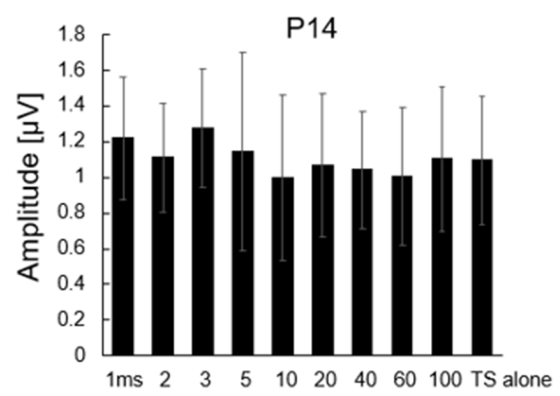

D
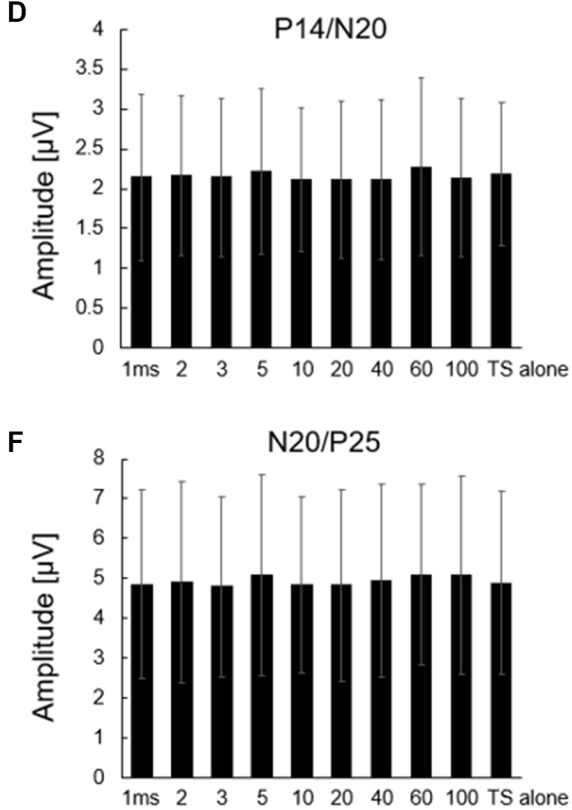

FIGURE 2 | Effect of CS to the right median nerve on P14, P14/N20, and N20/P25 responses of CP3 and CP4 electrodes. Averaged (A,B) P14, (C,D) P14/N20, and (E,F) N20/P25 amplitudes for TS alone and all ISI conditions (1, 2, 3, 5, 10, 20, 40, 60, and 100 ms). Left panels: CP3 electrode; right panels: CP4 electrode. No significant differences were observed between each ISI condition and TS alone condition in all amplitudes. All data are presented as means \pm SD.

If a direct connection between bilateral ascending pathways exists, the amplitude of SEP induced by the second median nerve TS would be attenuated or eliminated by the refractory period under the short ISI condition (Hoshiyama and Kakigi, 2002). However, in our result, the right median nerve stimulation (CS) did not decrease the cortical and subcortical components of the SEP induced by the left median nerve TS in short ISI conditions (1, 2, 3, and $5 \mathrm{~ms})$. This result suggests the absence of a neural connectivity formed by a small number of synapses in the left-right interaction of the somatosensory pathway.

Regarding our results in the middle ISI condition (10-40 ms), Ragert et al. showed decreased N20 SEP amplitude with an ISI of 20-25 ms (Ragert et al., 2011). In contrast, Brodie et al. showed decreased P14/N20 SEP amplitude with an ISI of 25-35 ms and decreased N20/P25 SEP amplitude with an ISI of 15-35 ms, respectively (Brodie et al., 2014). In the present study, the right median nerve stimulation (CS) did not interfere with SEPs induced by the left median nerve stimulation (TS) in mid-length ISI conditions $(10,20$, and $40 \mathrm{~ms})$. This finding is inconsistent with the results of the two previous studies, and there is no consensus on the ISI interval regarding the left-right interaction of somatosensory pathways. However, the number of trials that were averaged was different between the previous studies and the current study (Ragert et al., 150 epochs; Brodie et al., 300 epochs; current study, 500 epochs). Importantly, increasing the number of SEP recordings improves the signal-to-noise ratio, suggesting that we were able to record SEPs by median nerve stimulation with a higher signal-to-noise ratio within the measurement conditions of the current study. Whether differences in the order of stimulation to the median nerve (Ragert et al., left to right; Brodie et al., right to left; current study, right to left) may account for conflicting results remains unclear.

The commissural fibers between the left and right hemispheres comprise not only the corpus callosum but 
also other commissural fibers such as the anterior and posterior commissural fibers. To investigate the left-right interaction of somatosensory pathways by polysynaptic connections, we included long ISIs (60 and $100 \mathrm{~ms}$ ) in our evaluations and found no significant inhibitory effects of the first stimulation to the right median nerve on the SEP evoked by the second stimulation to the left median nerve. This finding suggests that there may not be a pathway to input to the contralateral SI over a long period of time or that investigating the long left-right connectivity by polysynaptic connections might be difficult using the p-SEP method.

A study with cortical surface SEP recordings in patients undergoing epileptogenic tissue resection revealed that four of 41 patients showed ipsilateral SEPs, which were very localized, were of considerably lower amplitude, had a longer latency (1.2-17.8 ms), and did not show an initial negativity (Noachtar et al., 1997). The uncrossed ipsilateral afferent system from the upper extremities includes the spinoreticular and spinomesencephalic tract, which may reach the ipsilateral somatosensory cortex (Scheibel, 1984; Noachtar et al., 1997). Although any changes in SEPs could not be identified with the electrode ipsilateral to the median nerve TS, somatosensory information may actually be directly inputted to the ipsilateral cerebrum. The existence of pathways that transfer somatosensory information to the contralateral hemisphere without involving the corpus callosum is important for recovery from hypoesthesia because when somatosensory pathways are damaged at the subcortical level, such as the thalamus, left-right interaction through the corpus callosum does not contribute to the recovery of hypoesthesia. Therefore, the presence of ipsilateral ascending pathways, which are very localized and have considerably lower amplitude using a multi-channel electrode in healthy adults, should be investigated. They should also be investigated in stroke patients, in whom large-scale changes in neural networks occur.

This study has several limitations. First, we performed a total of 5, 500 stimuli to the median nerve, which may have affected the activation or inhibition of somatosensory pathways. However, to minimize the order effect, each ISI condition was randomized. Second, all participants were men. The structure and function of the brain differs between men and women (Zaidi, 2010). Therefore, future

\section{REFERENCES}

Barbay, S., Guggenmos, D. J., Nishibe, M., and Nudo, R. J. (2013). Motor representations in the intact hemisphere of the rat are reduced after repetitive training of the impaired forelimb. Neurorehabil. Neural Repair 27, 381-384. doi: 10.1177/1545968312465193

Brodie, S. M., Villamayor, A., Borich, M. R., and Boyd, L. A. (2014). Exploring the specific time course of interhemispheric inhibition between the human primary sensory cortices. J. Neurophysiol. 112, 1470-1476. doi: 10.1152/jn.00 074.2014

Courtine, G., Roy, R. R., and Raven, J. (2005). Performance of locomotion and foot grasping following a unilateral thoracic corticospinal tract lesion in monkeys (Macaca mulatta) . Brain 128,2338-2358. doi: 10.1093/brain/awh604

Cruccu, G., Aminoff, M. J., Curio, G., Guerit, J. M., Kakigi, R., Mauguiere, F., et al. (2008). Recommendations for the clinical use of somatosensory-evoked studies should investigate sex differences in the interaction between contralateral and ipsilateral activations. Finally, the CS and TS were set to the same intensity and it remains possible that CS was not strong enough to affect the ipsilateral hemisphere. Future studies will include different CS intensities.

\section{DATA AVAILABILITY STATEMENT}

The original contributions presented in the study are included in the article/Supplementary Material, further inquiries can be directed to the corresponding author.

\section{ETHICS STATEMENT}

The studies involving human participants were reviewed and approved by the Ibaraki Prefectural University of Health Sciences Review Board (approval no. 893 and e278). The patients/participants provided their written informed consent to participate in this study.

\section{AUTHOR CONTRIBUTIONS}

Conceptualization: DI, KI, HY, KT, AY, and YKo. Methodology and investigation: DI, KI, HY, and YKa. Formal analysis: DI, KT, and SY. Writing the original draft: DI. Review and editing of manuscript: KI, HY, KT, SY, YKa, AY, and YKo. All authors contributed to the article and approved the submitted version.

\section{FUNDING}

This work was supported by JSPS KAKENHI (Nos. $18 \mathrm{~K} 17725$ and $21 \mathrm{H} 03305$ to DI; and No. $19 \mathrm{H} 05730$ to AY) and a Grant-in-Aid for Project Research (No. 1962-1 to YKo) from the Ibaraki Prefectural University of Health Sciences.

\section{SUPPLEMENTARY MATERIAL}

The Supplementary Material for this article can be found online at: https://www.frontiersin.org/articles/10.3389/fnsys.2021.6987 58/full\#supplementary-material.

potentials. Clin. Neurophysiol. 119, 1705-1719. doi: 10.1016/j.clinph.2008 03.016

Faul, F., Erdfelder, E., Lang, A. G., and Buchner, A. (2007). G*Power 3: a flexible statistical power analysis program for the social, behavioral and biomedical sciences. Behav. Res. Methods 39, 175-191. doi: 10.3758/bf03193146

Frost, S. B., Barbay, S., Friel, K. M., Plautz, E. J., and Nudo, R. J. (2003). Reorganization of remote cortical regions after ischemic brain injury: a potential substrate for stroke recovery. J. Neurophysiol. 89, 3205-3214. doi: 10.1152/jn.01143.2002

Hoshiyama, M., and Kakigi, R. (2002). New concept for the recovery function of short-latency somatosensory evoked cortical potentials following median nerve stimulation. Clin. Neurophysiol. 113, 535-541. doi: 10.1016/s13882457(02)00039-1

Isa, T. (2017). The brain is needed to cure spinal cord injury. Trends Neurosci. 40, 625-636. doi: 10.1016/j.tins.2017.08.002 
Isa, T. (2019). Dexterous hand movements and their recovery after central nervous system injury. Annu. Rev. Neurosci. 42, 315-335. doi: 10.1146/annurev-neuro070918-050436

Isa, T., Mitsuhashi, M., and Yamaguchi, R. (2019). Alternative routes for recovery of hand functions after corticospinal tract injury in primates and rodents. Curr. Opin. Neurol. 32, 836-843. doi: 10.1097/WCO.0000000000000749

Iwamura, Y., Iriki, A., and Tanaka, M. (1994). Bilateral hand representation in the postcentral somatosensory cortex. Nature 369, 554-556. doi: 10.1038/369554a0

Iwamura, Y., Tanaka, M., Iriki, A., Taoka, M., and Toda, T. (2002). Processing of tactile and kinesthetic signals from bilateral sides of the body in the postcentral gyrus of awake monkeys. Behav. Brain Res. 135, 185-190. doi: 10.1016/s01664328(02)00164-X

Iwamura, Y., Taoka, M., and Iriki, A. (2001). Bilateral activity and callosal connections in the somatosensory cortex. Neuroscientist 7, 419-429. doi: 10.1177/107385840100700511

Kato, J., Yamada, T., Kawaguchi, H., Matsuda, K., and Higo, N. (2020). Functional near-infrared-spectroscopy-based measurement of changes in cortical activity in macaques during post-infarct recovery of manual dexterity. Sci. Rep. 10:6458. doi: 10.1038/s41598-020-63617-0

Levy, C. E., Nichols, D. S., Schmalbrock, P. M., Keller, P., and Chakeres, D. W. (2001). Functional MRI evidence of cortical reorganization in upper-limb stroke hemiplegia treated with constraint-induced movement therapy. Am. J. Phys. Med. Rehabil. 80, 4-12. doi: 10.1097/00002060-200101000-00003

Mauguiere, F., Allison, T., Babiloni, C., Buchner, H., Eisen, A. A., Goodin, D. S., et al. (1999). Somatosensory evoked potentials. The international federation of clinical neurophysiology. Electroencephalogr. Clin. Neurophysiol. 52, 79-90.

Murata, Y., Higo, N., Hayashi, T., Nishimura, Y., Sugiyama, Y., Oishi, T., et al. (2015). Temporal plasticity involved in recovery from manual dexterity deficit after motor cortex lesion in macaque monkeys. J. Neurosci. 35, 84-95. doi: 10.1523/JNEUROSCI.1737-14.2015

Netz, J., Lammers, T., and Homberg, V. (1997). Reorganization of motor output in the non-affected hemisphere after stroke. Brain 120, 1579-1586. doi: 10.1093/brain/120.9.1579

Nihashi, T., Naganawa, S., Sato, C., Kawai, H., Nakamura, T., Fukatsu, H., et al. (2005). Contralateral and ipsilateral responses in primary somatosensory cortex following electrical median nerve stimulation-an fMRI study. Clin. Neurophysiol. 116, 842-848. doi: 10.1016/j.clinph.2004.10.011

Noachtar, S., Luders, H. O., Dinner, D. S., and Klem, G. (1997). Ipsilateral median somatosensory evoked potentials recorded from human somatosensory cortex. Electroencephalogr. Clin. Neurophysiol. 104, 189-198. doi: 10.1016/s01685597(97)00013-0

Nudo, R. J., Wise, B. M., SiFuentes, F., and Milliken, G. W. (1996). Neural substrates for the effects of rehabilitative training on motor recovery after ischemic infarct. Science 272, 1791-1794. doi: 10.1126/science.272.52 69.1791

Ragert, P., Nierhaus, T., Cohen, L. G., and Villringer, A. (2011). Interhemispheric interactions between the human primary somatosensory cortices. PLoS One 6:e16150. doi: 10.1371/journal.pone.0016150

Ramanathan, D., Conner, J. M., and Tuszynski, M. H. (2006). A form of motor cortical plasticity that correlates with recovery of function after brain injury. Proc. Natl. Acad. Sci. U S A 103, 11370-11375. doi: 10.1073/pnas.0601 065103

Rosenzweig, E. S., Courtine, G., Jindrich, D. L., Brock, J. H., Ferguson, A. R., Strand, S. C., et al. (2010). Extensive spontaneous plasticity of corticospinal projections after primate spinal cord injury. Nat. Neurosci. 13, 1505-1510. doi: $10.1038 / \mathrm{nn} .2691$

Scheibel, A. B. (1984). The Brain Stem Reticular Core and Sensory Function. Bethesda, MD: American Physiology Society.

Yamamoto, T., Hayashi, T., Murata, Y., Ose, T., and Higo, N. (2019). Premotor cortical-cerebellar reorganization in a macaque model of primary motor cortical lesion and recovery. J. Neurosci. 39, 8484-8496. doi: 10.1523/JNEUROSCI.0077-19.2019

Yoshino-Saito, K., Nishimura, Y., Oishi, T., and Isa, T. (2010). Quantitative intersegmental and inter-laminar comparison of corticospinal projections from the forelimb area of the primary motor cortex of macaque monkeys. Neuroscience 171, 1164-1179. doi: 10.1016/j.neuroscience.2010.10.007

Zaidi, Z. F. (2010). Gender differences in human brain: a review. Open Anat. J. 2, 37-55. doi: 10.2174/1877609401002010037

Conflict of Interest: The authors declare that the research was conducted in the absence of any commercial or financial relationships that could be construed as a potential conflict of interest.

Publisher's Note: All claims expressed in this article are solely those of the authors and do not necessarily represent those of their affiliated organizations, or those of the publisher, the editors and the reviewers. Any product that may be evaluated in this article, or claim that may be made by its manufacturer, is not guaranteed or endorsed by the publisher.

Copyright (c) 2021 Ishii, Ishibashi, Yuine, Takeda, Yamamoto, Kaku, Yozu and Kohno. This is an open-access article distributed under the terms of the Creative Commons Attribution License (CC BY). The use, distribution or reproduction in other forums is permitted, provided the original author(s) and the copyright owner(s) are credited and that the original publication in this journal is cited, in accordance with accepted academic practice. No use, distribution or reproduction is permitted which does not comply with these terms. 